\title{
Outcome of Percutaneous Nephrolithotomy in the Management of Lower Pole Stones
}

\author{
Udaya Man Singh Dongol, ${ }^{1}$ Sandeep Bohora ${ }^{1}$ \\ ${ }^{1}$ Department of Urosurgery, Kathmandu Medical College and Teaching Hospital, Kathmandu.
}

\section{ABSTRACT}

Background: Urolithiasis is a worldwide problem due to its high prevalence and recurrence. Percutaneous
nephrolithotomy is a minimally invasive surgical option for the treatment of large renal stone burden greater than
$20 \mathrm{~mm}$, staghorn calculi and lower pole calyceal stone greater than $10 \mathrm{~mm}$. The objective of this study was to evaluate
the safety and efficacy of percutaneous nephrolithotomy in the management of lower pole calyceal stones.
Methods: Seventy patients who presented in between June 2013 and September 2017 with lower pole calyceal
stones and lower calyceal stones with pelvic extension were included in the study. The operating time, the hospital stay,
complications rate, stone clearance rate were all noted. Patients were followed up in three and six weeks with X-ray
KUB and ultrasonography of abdomen.
Results: Seventy adult patients with lower pole calyceal stones underwent standard percutaneous nephrolithotomy.
The mean age was 32 years ( $18-71$ yrs). The mean stone size was 17.6 mm (15 - 28 mm). The mean operating time
was 62 minutes ( $48-124$ mins) and hospital stay was 4.1 days(4-8 days). The stone clearance rate was $92.6 \%$ for stone
$<20$ mm and $90.7 \%$ stone size $>20$ mm. The complications noted were fever ( $8.5 \%)$, transient haematuria $(20 \%)$,
urine leak (5.7\%), obstruction by residual fragments ( $5.7 \%)$ and one pseudoaneurysm(1.42\%). Seven patients $(10 \%)$
needed blood transfusion.
Conclusions: Percutaneous nephrolithotomy is a safe, feasible and highly effective method for the treatment of lower
pole calyceal stones.
Keywords: Lower calyx; lower pole stones; percutaneous nephrolithotomy; stone free rate.

INTRODUCTION

Urolithiasis makes significant health concern for large population. ${ }^{1}$ Lower pole stones make approximately 25 $30 \%$ of renal stones bulk. ${ }^{2}$ The incidence of renal stones is increasing but the ideal treatment of lower pole stone remains controversial regarding extracorporeal lithotripsy (ESWL), percutaneous nephrolithotomy (PCNL) and flexible ureteroscopy(RIRS). ${ }^{3,4}$ The effectiveness of these techniques are determined by size, composition of stone and spatial anatomy of lower pole. ${ }^{5-7}$ Percutaneous nephrolithotomy is effective and is preferred to ESWL for stones larger than $20 \mathrm{~mm} .{ }^{8}$ The success of ESWL is related to size and composition of stone and anatomic features of lower pole. ${ }^{9}$ Flexible ureteroscopy can be considered for stones less than $20 \mathrm{~mm}$ in obese patients with complicated intra-renal anatomy and hard stones. ${ }^{10}$ This study has been conducted to evaluate the efficacy and safety of PCNL in the management of lower pole stones. METHODS

This was a prospective observational study. Seventy adult patients treated with PCNL in between June 2013 and September 2017 for lower calyceal stones and stones in lower calyx with pelvic extension were studied in the Department of Urosurgery, Kathmandu Medical College and Teaching Hospital. The patients with stones in other calyces of the kidney including only pelvic, upper calyceal, upper ureteral and complete staghorn calculus were excluded from the study. The treatment modalities of ESWL and RIRS were not available in our institute. These options were well explained and offered to the patients before we proceeded with PCNL. MiniPCNL was also not available in our centre at that time. Ethical approval was taken from the ethical committee and the written informed consent was taken from all patients.

All the patients were evaluated with complete urine analysis, urine culture and sensitivity, complete haemogram, coagulation profile, renal function tests, ultrasonography of abdomen, intravenous urography. Positive cultures were treated with antibiotics. All patients were admitted one day before the procedure and prophylactic intravenous antibiotic was started on
DOI: http://dx.doi.org/10.3126/

inhrc.v16i3.21423
Correspondence: Udaya Man Singh Dongol, Department of Urosurgery, Kathmandu Medical College and Teaching Hospital, Sinamangal, Kathmandu Nepal. Email: dongoludaya@gmail.com, Phone: +9779851053217. 
the same day of surgery at the time of induction of general anaesthesia.

Standard PCNL was performed in prone position for all patients under general anesthesia by the consultant. After insertion of $6 \mathrm{Fr}$. ureteral catheter and delineation of pelvicalyceal system with contrast media, lower calyx and / or middle calyx puncture was done under fluoroscopic guidance with $18 \mathrm{G}$ angiography needle and Terumo guide wire $(0.035,, 150 \mathrm{~cm})$ inserted advancing to the calyx, renal pelvis and secured to the ureter. Acute dilatation of tract was done up to $22 \mathrm{Fr}$ with metallic coaxial dilators. Then nephrolithotripsy was performed with 20Fr nephroscope(®Wolf) and pneumatic lithotripter (®Nidhi).

All the stone fragments were removed by grasping forcep. A $20 \mathrm{Fr}$ nephrostomy tube was inserted after antegrade placement of $6 \mathrm{Fr}$ double $\mathrm{J}$ stent as the standard practice of conventional PCNL. Mini-PCNL was not available in our institute at the time of this study. The operating time, complications rate, hospital stay, stone clearance rate were all recorded.The patients with residual fragments less than $4 \mathrm{~mm}$ were considered stone free.Ultrasonography of abdomen and pelvis was done to measure the size of these stones in three weeks follow up. Nephrostomy tube was removed on third postoperative day in the patients with significant intraoperative bleeding and suspected breach of pelvicalyceal system. In other patients it was removed on second postoperative day early in the morning. DJ stent was removed in three weeks. Patients were followed up in three and six weeks by X-ray KUB and ultrasonography of abdomen.

Data were collected and statistically analysed using Statistical Package for the Social Sciences, SPSS version 20. Descriptive statistics were used. Chi-Square test was applied to see the stone free rate in relation with the stone burden.

\section{RESULTS}

Seventy patients with lower calyceal stone and lower calyceal stones with extension to the pelvis underwent standard PCNL. There were 42 male patients and 28 female patients with the mean age of 32 years (18 to 71 years). The stone were located on left side in 36 patients and on right side in 34 patients. The mean size of stone was $17.6 \mathrm{~mm}$ (15-28mm). Twenty three patients had mild hydronephrosis and moderate hydronephrosis was seen in nine patients due to partial extension of stone into the pelvis. The hydronephrosis in these patients could also be sequelae of previous surgery or the previous residual hydronephrosis. Lower pole focal caliectasis was also seen in 32 patients. There was no hydronephrosis in rest of the patients.

Ultrasonography was used to measure the size of these stones. Twelve patients had undergone open surgery before. The mean operating time from puncture to completion of procedure was 62 minutes (48-124 $\mathrm{min})$. Stone removal was successful through lower pole puncture in 48 patients and through middle calyx puncture in 22 patients. The stone free rate for stone larger than $2 \mathrm{~cm}$ was $90.7 \%$ and for stone less than 2 $\mathrm{cm}$, it was $92.6 \%$. The patients with residual stones were later treated with ESWL.

The complications encountered were mostly of Clavien grade 2 . One patient $(1.42 \%)$ presented with massive haematuria on eleventh post-operative day and was found to have pseudoaneurysm (Clavien grade $3 \mathrm{~b}$ ) for which coil angioembolization was done. Transient haematuria was seen in $14(20 \%)$ patients. However, seven patients(10\%) needed blood transfusion. The preoperative mean haemoglobin level of these seven patients was $10.1 \mathrm{gm} \%$ which dropped to mean of 8.0 gm\%. Blood transfusion was done on the following day after haemoglobin report and after clinical assessment of these patients. With mini-PCNL, the transfusion rate could be expected to decrease. However the mean postoperative haemoglobin drop after 24 hours was only $0.76 \mathrm{gm} \%$ of the average haemoglobin of $12.4 \mathrm{gm} \%$. Fever was seen in $6(8.5 \%)$ patients and was managed with antibiotics according to the urine culture report and DJ stent was removed at their earliest. Four patients had prolonged urine leak after removal of nephrostomy tube. In eight patients(11.4\%), clinically insignificant residual fragments of stones $<4 \mathrm{~mm}$ were seen. In four patients $(5.7 \%)$, there was obstruction of kidney by these small stones after removal of DJ stent. They were found to pass these fragments spontaneously in next follow up.

Mean hospitalization was 4.1 days (4-8 days). In six weeks follow up, the stone free rate was $92.6 \%$ for stone size $<20 \mathrm{~mm}$ and $90.7 \%$ for stone $>20 \mathrm{~mm}$.

Table 1. Demographic variables of patients.

\begin{tabular}{lr} 
Total & Number of patients(\%) \\
\hline Sex & \\
Male & $42(60 \%)$ \\
Female & $28(40 \%)$
\end{tabular}

Site of stone

$\begin{array}{lr}\text { right side } & 34(48.5 \%) \\ \text { left side } & 36(51.5 \%) \\ \text { Mean age (Years) } & 32(18-71) \\ \text { Mean stone size } \mathrm{mm} & 17.6\end{array}$




\begin{tabular}{|c|c|c|c|}
\hline \multicolumn{3}{|l|}{ Stone size range $\mathrm{mm}$} & $15-28$ \\
\hline $\begin{array}{l}\text { Table } 2 \text {. Outcome } \\
\text { postoperative varia }\end{array}$ & s of per & dute dilu & \\
\hline Stone size & $\begin{array}{l}\text { Number of } \\
\text { patients(\%) }\end{array}$ & $\begin{array}{l}\text { Stone } \\
\text { free rate }\end{array}$ & $\begin{array}{r}P \\
\text { value }\end{array}$ \\
\hline $15-20 \mathrm{~mm}$ & $27(38.5 \%)$ & $92.6 \%$ & 0.78 \\
\hline$>20 \mathrm{~mm}$ & $43(61.5 \%)$ & $90.7 \%$ & \\
\hline $\begin{array}{l}\text { Ancillary } \\
\text { procedure(ESWL) }\end{array}$ & $6(8.5 \%)$ & & \\
\hline $\begin{array}{l}\text { Operation } \\
\text { time mean, mins }\end{array}$ & $62(48-124)$ & & \\
\hline $\begin{array}{l}\text { Hospitalization } \\
\text { (mean , days ) }\end{array}$ & $4.1(4-8)$ & & \\
\hline
\end{tabular}

\begin{tabular}{lr}
\hline Table 3. Complications. & \\
\hline Transient haemorrhage & $14(20 \%)$ \\
\hline Fever & $6(8.5 \%)$ \\
\hline Obstruction & $4(5.7 \%)$ \\
\hline Blood transfusion & $7(10 \%)$ \\
\hline Urine leak & $4(5.7 \%)$ \\
\hline Pseudoaneurysm & $1(1.42 \%)$ \\
\hline
\end{tabular}

\section{DISCUSSION}

Despite the significant amount of data reported, the indications of PCNL, ESWL and recently flexible ureteroscopy(RIRS) for the treatment of lower calyceal stone still remain unsettled. ${ }^{11} \mathrm{ESWL}$ is usually the first choice of treatment by majority of patients because of good patient tolerance, low complication rate, low cost and less hospital stay. But however, it is associated with low success rate and higher retreatment is required. ${ }^{12}$ The infundibulopelvic angle, infundibular length and width are major anatomical factors in the stone clearance in ESWL. ${ }^{5}$ On the contrary, PCNL is not affected by any of these factors. ${ }^{13}$ RIRS with newer digital flexible ureteroscopes is being preferred as treatment modality for low volume renal stone diseases recently. But the success rate of flexible ureteroscopy is significantly low in unfavourable anatomical conditions such as long and narrow lower calyceal infundibulum and acute infundibulopelvic angle. ${ }^{14}$

Percutaneous nephrolithotomy constitutes the first line therapy for large renal stone burden including renal calculi greater than $20 \mathrm{~mm}$, lower pole calyceal stones greater than $10 \mathrm{~mm}$ and upper ureteral stones. ${ }^{15}$ It has been proved to be a less morbid procedure compared to open stone surgery and better stone clearance than ESWL. ${ }^{16}$ The recent guidelines of European Association of Urology(EAU) also recommend PCNL for lower pole calyceal stones $>1.5 \mathrm{~cm}$. For smaller lower pole calyceal stones, ESWL is recommended if lithotripsy resistant hard stones such as brushite and cystine stones, long and narrow infundibulum, are absent. PCNL is otherwise considered a good and reasonable alternative. ${ }^{17}$ In this study, however, the outcome of PCNL in the management of lower calyceal stone has been reported and compared to the reports of other methods.

Since the introduction of PCNL in 1976 by Fernstrom and Johansson, with the marked improvement in techniques and instruments, PCNL is being used for the treatment of renal stone diseases with highest stone free rate. ${ }^{17}$ Despite its slight invasiveness, of all the treatment options, PCNL delivers the stone free rate more than $90 \%$ for lower pole stones $>20 \mathrm{~mm} .{ }^{18-21}$

In the present study, the overall stone free rate for PCNL in six weeks was $92.6 \%$ for stone size less than $20 \mathrm{~mm}$ and $90.7 \%$ for stone size $>20 \mathrm{~mm}$ which was comparable to the stone free rate in the series by Netto et al and Cass AS. ${ }^{22,23}$ The study also showed that the stone free rate in relation to stone size was not statistically significant ( $P$ value 0.78 ). In the multicentric prospective randomised trial by Albala et al, it was also found that stone free rate in PCNL for lower pole stones was not dependent on the size of stones. ${ }^{24}$ Six patients(8.5\%) needed ancillary procedure in the form of ESWL for the stone clearance. In the study by Haroon et al, six patients $(10.6 \%)$ needed ancillary procedures ( five ESWL and one Double $\mathrm{J}$ placement). ${ }^{25}$ Similarly the study by Pardalidis et al ${ }^{26}$ and Havel and colleagues ${ }^{27}$ showed stone free rate for stone $>20 \mathrm{~mm}$ to be $93.7 \%$.

In our study, the complications encountered were mostly of Clavien grade 2 . One patient (1.42\%) presented with massive haematuria on eleventh day due to pseudoaneurysm for which coil angioembolization was successfully done.The other complications included fever in six $(8.5 \%)$, ureteral obstruction in four $(5.7 \%)$ patients. Seven patients $(10 \%)$ needed blood transfusion which was done on the second postoperative day after haemoglobin report as well as after clinical assessment of these patients. The blood transfusion rate could have been lowered with Mini-PCNL. In four patients(5.7\%), prolonged urine leak up to fourth day after removal of nephrostomy tube was seen. The overall complications rate in the study by Haroon et al was 19\% including one urosepsis and one pseudoaneurysm requiring angioembolization. ${ }^{25}$ In the UK Health Statistics database reviewed for more than 5700 patients undergoing PCNL over the period of six years, the complications of haemorrhage(1.4\%), urinary tract infection(3.8\%), fever $(0.7 \%)$, and 30 -day readmission(9\%) were seen. ${ }^{28}$ Similarly, Pan et al reported the overall complications rate of $11.86 \% .{ }^{29}$ In the series of 107 patients by Raut 
Percutaneous Nephrolithotomy in the Management of Lower Pole Stones

NK et al, the complications of urinary tract infection was seen in $12.1 \%$, urine leak in $4.6 \%$, urosepsis in $1 \%$ and uncomplicated fever in $71 \%$. The overall stone free rate was $85.98 \%$ in their series. Thirteen patients with residual stones underwent ESWL while relook pcnl was done in one patient and ureteroscopy in one patient. ${ }^{30}$

In our study, the mean operative time was 62 minutes and the mean hospital stay was 4.1 days. The operative time and hospital stay was comparable with the findings of the series by Pan et al. ${ }^{29}$ However, the hospital stay was comparatively much shorter in few other studies. ${ }^{31,32}$ In our study, we admitted all the patients one day before surgery and the nephrostomy tube drain was removed on the third postoperative day in those patients with significant intraoperative bleeding and suspected breach of pelvicalyceal system.

The limitation of our study is that the Hounsfield measurement to assess the density of the stones was lacking and therefore the density was not measured. We followed our patients with X-ray KUB and ultrasonography in the postoperative period which might have lowered our ability to detect the residual fragments. According to the study by Denstedt et al, up to $35 \%$ of the residual stones can be missed in plain film. ${ }^{33} \mathrm{ACT}$ scan is the most sensitive method to detect the residual fragments. ${ }^{34}$

\section{CONCLUSIONS}

PCNL is a safe, feasible and highly effective method for the treatment of lower pole calyceal stones.

\section{REFERENCES}

1. Hartman C, Gupta N, Leavitt D, Hoenig D, Okeke Z, Smith A. Advances in percutaneous stone surgery. Asian J Urol. 2015;2(1):26-32.[Science Direct]

2. Moore SL, Bres-Niewada E, Cook P, Wells H, Somani BK. Optimal management of lower pole stones: the direction of future travel. Cent European J Urol. 2016;69(3):274. [PubMed]

3. Kim BS. How to determine the treatment options for lower-pole renal stones. Annals Transl Med. 2016;4(16). [PubMed]

4. Knoll T, Buchholz N, Wendt-Nordahl. Extracorporeal shockwave lithotripsy versus Percutaneous nephrolithotomy versus flexible ureterorenoscopy for lower-pole stones. Arab J Urol. 2012;10:336-41.

5. Lingerman JE, Siegel YL, Steele B, Nyhuis AW, Woods JR. Management of lower pole nephrolithiasis. A critical analysis. J Urol. 1994;151:663-67.[DOI]

6. Conort P, Dore B, Saussine C. Guidelines for the urological management of renal and ureteric stones in adults. Prog Urol. 2004;14(6):1095-102.[Europe PMC]

7. Sumino Y, Mimata H, Tasaki Y, Ohno H, Hoshino T. Predictors of lower pole renal stone clearance after extracorporeal shock wave lithotripsy. J Urol.2002;168:1344-47. [Science Direct]

8. May DJ, Chandhoke PS. Efficacy and cost-effectiveness of extracorporeal shock wave lithotripsy for solitary lower pole renal calculi. J Urol. 1998;159(1):24-7.[Science Direct]

9. Khan M, Lal M, Kash DP, Hussain M, Rizvi S. Anatomical factors predicting lower calyceal stone clearance after extracorporeal shockwave lithotripsy. Afr J Urol. 2016;22(2):96-100.[Full Text Link]

10. Ziaei S, Nasehi A, Sasiri A, Simforooshi N, Danesh AK, Sharifi AF et al. PCNL in the management of lower Pole calyceal calculi .Urol J. 2004:3:174-76.

11. Abbott JE, DiMatteo AD, Fazio E, Deem SG, Sobh AK, DePolo A, Davalos JG. High supracostal percutaneous nephrolithotomy access: Assessing safety in access above the eleventh rib after performing preoperative planning with computed tomography. Open J Urol. 2015;5(04):25. [Google Scholar]

12. Kruck S, Anastasiadis AG, Herrmann TR . Minimally invasive percutaneous nephrolithotomy: an alternative to retrograde intrarenal surgery and shockwave lithotripsy. World J Urology. 2013;31:1555-61.[Link]

13. Elbahnasy AM, Shalhav AL, Hoenig DM, Elashry OM, Smith DS, Mcdougall EM, Clayman RV. Lower caliceal stone clearance after shock wave lithotripsy or ureteroscopy: the impact of lower pole radiographic anatomy. J Urol. 1998;159(3):676-82. [Science Direct]

14. Shah K, Agrawal MS, Mishra DK. Superperc: A new technique in minimally-invasive percutaneous nephrolithotomy. Indian J Urol. 2017;33(1):48.[PubMed]

15. Feng MI, Tamaddon K, Mikhail A, Kaptain JS, Bellman GC. Prospective randomized study of various techniques of percutaneous nephrolithotomy. Urology. 2001;58:34550.[Science Direct]

16. Shrivastava A and Chipde SS. Management of 1-2 cm renal stones. Indian J Urol. 2013;29(3)195-99.[PubMed]

17. EI-Nahas A, Eraky I, Shokeir AA, Shoma AM, EI-Assmy AM, EI-Tabey NA et al. Percutaneous nephrolithotomy for treating staghorn stones: 10 years of experience of a tertiary-care centre. Arab J Urol. 2012;10:324-29. [Science Direct]

18. Donaldson JF, Lardas M, Scrimgeour D. Systematic 
review and meta-analysis of the clinical effectiveness of shockwave lithotripsy, retrograde intrarenal surgery and percutaneous nephrolithotomy for lower-pole renal stones.Eur Urol.2015; 67:612-16.[Science Direct]

19. Koyuncu H, Yencilek F, Kaikan M, Bastug Y, Yencilek E, Odemir AT et al. Intrarenal surgery versus percutaneous nephrolithotomy in the management of lower pole stones greater than $2 \mathrm{~cm}$. IntBraz J Urol. 2015;41:245-51.[Full Text Link]

20. Lee SW, Chaiyakunapruk N, Chong HY, Liong ML. Comparative effectiveness and safety of various treatment procedures for lower pole renal calculi: A systematic review and network meta-analysis. BJU Int. 2015;116:252-64. [Full Text Link]

21. De la Rosette J, Assimos D, Desai M, Gutierrez J, Lingeman J, Scarpa R et al. The clinical research office of the endourological society percutaneous nephrolithotomy global study: indications,complications and outcomes in 5803 patients. J Endourol. 2011;25:11-17.[DOI]

22. Netto NRJ, Claro JF, Lexmos GC, Cortado PL. Renal Calculi in lower pole calices. What is the best method of treatment ? J Urol.1991;146:721-23. [Science Direct]

23. Cass AS . Extracorporeal shock wave lithotripsy or Percutaneous Nephrolithotripsy for lower pole lithiasis? J Endourol .1996;10:17-20.[Full Text Link]

24. Albala DM, Assimos DG. Prospective randomized trial of extracorporeal shock wave lithotripsy and percutaneous nephrolithotomy for lower pole nephrolithiasis; initial results .J Urol. 2001; 166:2072-80.[Science Direct]

25. Haroon N, Naim SM, HammadAther M. Optimal Management of Lower Pole Calyceal stone 15 to $20 \mathrm{~mm}$. Korean J Urol. 2013;54:258-62.[Full Text Link]

26. Pardalidis NP, Andripocelos NA, Sountoulidis P, Kosmaogloce EV. Should percutaneous nephrolithotripsy be considered the primary therapy for lower pole stone? J Endourol. 2010;24:219-22.[DOI]
27. Havel D, Saussine C, Fath C, Lang H ,Faure F, Jacqmin D et al. Single stone of lower pole of kidney .Comparative results of ESWL and PCNL. Euro Urol.1998; 33:396400.[Full Text Link]

28. Armitage JN, Withington J, Van der MeulenJ . Percutaneous nephrolithotomy in England: practice and outcomes described in the Hospital Episode Statistics database. BJU Int. 2014;113:777-82.[DOI]

29. Pan J,Chen Q, Xue W. RIRS versus PCNL for single renal stone of $2-3 \mathrm{~cm}$ : Clinical outcome and cost effective analysis in Chinese medical setting. Urolithiasis.2013;41:73-78. [Full Text Link]

30. Raut NK, Singhania P, Joshi N, Shringarpure S, Sathe S, Tiwari N. Prospective study of Percutaneous Nephrolithotomy in the Management of renal calculi. MGM J Med Sci. 2017;4(1):1-5.

31. Kirac M, Bozkurt OF, Ync L, Guneri C, Unsal A, Biri H et al.Comparison of retrograde intrarenal surgery and percutaneous nephrolithotomy in the management of lower pole renal stones with a diameter of smaller than 15 mm. Urolithiasis. 2013;4:241-6.

32. Sabnis RB, Ganesamoni R, Doshi A, Ganapule AP, Jagtap J, Desai MR et al. Percutaneous nephrolithotomy versus retrograde intrarenal surgery for the management of smaller renal calculi: A randomised controlled trial. BJU Int. 2013;112:355-61.

33. Denstedt JD, Clayman RV, Picus DD. Comparison of endoscopic and radiological residual fragment rate following percutaneousnephrolithotripsy. J Urol.1991;145:703-5.[Science Direct]

34. Gaucher O, Cormier L, Deneuville M, Regent D, Mangin $\mathrm{P}$, Hubert $\mathrm{J}$ et al. Which is the best performing imaging method for demonstrating residual renal calculi? Prog Urol. 1998;8:493-501.[Link] 